\title{
Evaluating the Number of Ligand Binding Sites on Protein from Tryptophan Fluorescence Quenching under Typical Experimental Conditions
}

\author{
Alexey V. Gayer ${ }^{1}$, Boris P. Yakimov ${ }^{1}$, Gleb S. Budylin², and Evgeny A. Shirshin ${ }^{1,3^{*}}$ \\ ${ }^{1}$ Department of Physics, M.V. Lomonosov Moscow State University, 1/2 Leninskie gory, Moscow 119991, Russia \\ ${ }^{2}$ National Research University Higher School of Economics, Faculty of Physics, 20 Myasnitskaya str., Moscow 101000, \\ Russia \\ ${ }^{3}$ Institute of spectroscopy of the Russian Academy of Sciences, 5 Fizicheskaya str., Moscow 108840, Russia \\ *e-mail: shirshin@lid.phys.msu.ru
}

\begin{abstract}
Fluorescence quenching technique is extensively applied for the characterization of intermolecular interactions in the solution that is one of the major problems in biochemistry and pharmacology. Using the SternVolmer equation, one can obtain a measure of binding affinity calculated under the assumption of static quenching, while the possibility to determine other binding parameters is under discussion. Several mathematical approaches are known, which allow determining the number of binding sites from fluorescence quenching curves. However, they usually require high concentrations of the ligand to obtain saturating binding curves that could be complicated in a number of experimental conditions. In this paper, we present a simple algorithm, which allows to prove that the number of binding sites in the system is equal to one or not and to verify that the quantum yield of the complex is zero. The advantage of the suggested approach is its applicability at typical conditions used in tryptophan fluorescence quenching experiments for the protein-ligand binding. A web interface for automated processing of fluorescence quenching experiments based on the suggested approach is presented. (C) 2020 Journal of Biomedical Photonics \& Engineering.
\end{abstract}

Keywords: fluorescence quenching; Stern-Volmer; tryptophan fluorescence quenching; protein-ligand binding; number of binding sites; drug discovery.

Paper \#3365 received 12 May 2020; accepted for publication 28 May 2020; published online 22 Jun 2020. doi: 10.18287/JBPE20.06.020303.

\section{Introduction}

Quantitative characterization of binding affinities is a central task of biochemistry and pharmacology $[1,2]$. It requires determination of binding sites number and affinity constants for each site. To obtain a binding curve, one should use a method, which is sensitive to a change of a certain physical parameter upon complex formation, e.g. diffusion coefficient, surface plasmon resonance properties, hydrodynamic radius, enthalpy etc. [3]. Among others, optical molecular spectroscopy techniques can be used, including the fluorescence quenching technique (FQT) - in cases when fluorescence is quenched upon binding [4]. In this paper, we will focus on the assessment of protein-ligand binding by analyzing intrinsic protein fluorescence quenching.

Since there is still a confusion in terminology concerning different types of quenching, we will first introduce it according to Ref. [5]. In the systems where fluorophore and quencher are different molecules, two types of quenching could be distinguished: collisional quenching (in which the diffusion is required to bring the quencher close enough to fluorophore) and bindingrelated (often called "static") quenching (for which quencher and fluorophore are bound, so that the 
quenching process occurs immediately upon excitation) [5]. The efficiency of binding-related quenching, characterized by the quenching constant, depends on the binding affinity, hence, by obtaining the dependence of the fluorescence intensity on the ligand concentration, one can determine binding parameters [4]. However, even under the assumption of binding-related (static) quenching, two distinct cases should be considered: the first is when the fluorescence of protein-ligand complex is negligible in comparison with the protein fluorescence (i.e. complete quenching), and the second when the fluorescence of protein-ligand complex is less, but comparable with the protein fluorescence (i.e. incomplete quenching). Obviously, affinity constants calculated based on fluorescence quenching parameters obtained in the case of incomplete quenching would significantly differ from the real affinity constant. Hence, understanding of fluorophore's quantum yield dependence on the occupied sites number is crucial for correct interpretation of the FQT data. Moreover, several papers point out the errors and pitfalls of FQT application for quantification of binding affinities and determination of the number of binding sites [6-10].

It should be noted that binding of a ligand to protein does not necessarily lead to quenching of fluorescence, and systems with the fluorescence enhancement are also known [11]. Indeed, the variation of fluorescence parameters upon binding ligands to proteins could be rather complex [12], however, in this paper we will focus on the most common case, when the emission intensity of tryptophan residues within a protein decreases upon ligand binding, i.e. fluorescence quenching is observed. A number of methods was suggested in the literature, which allow estimation of the number of binding sites from titration curves [1315]. Nevertheless, these methods require measurements of several titration curves in the regime when concentration of ligand must be high and significantly exceeds the characteristic dissociation constant of a complex [14-15], that is sometimes hardly possible due to experimental artifacts like the inner filter effect or low quantities of the ligand available. Hence, a simple and reliable test to assess the binding stoichiometry in more realistic experimental conditions is still of interest. In this paper, we suggest an algorithm for the assessment the binding parameters from fluorescence quenching curves, as well as a web-interface implementing this algorithm for on-line data processing.

\section{Backgrounds}

Consider the case of the 1:1 stoichiometry interaction:

$$
\mathrm{P}+\mathrm{Q} \rightleftarrows \mathrm{PQ}
$$

where P, Q and PQ correspond to protein, quencher and complex, respectively. Relation between equilibrium concentrations in this system can be expressed as

$$
\frac{[\mathrm{P}]_{\mathrm{T}}}{[\mathrm{P}]}=1+K_{\mathrm{A}}[\mathrm{Q}]
$$

where $[\mathrm{P}]_{\mathrm{T}},[\mathrm{P}]$ and $[\mathrm{Q}]$ are the total concentration of protein and equilibrium concentrations of protein and quencher, respectively, and $K_{\mathrm{A}}$ is the affinity (binding) constant. The Stern-Volmer equation is derived from (2) under the following assumptions:

(i) the concentration of complex is much less than quencher concentration ([PQ] $<<[\mathrm{Q}]$ ), thus yielding $[\mathrm{Q}] \approx[\mathrm{Q}]_{\mathrm{T}}$,

(ii) the quantum yield of the complex is approximately zero (complete quenching), thus fluorescence intensity $F$ is proportional to [P].

Under these assumptions Eq. (2) transforms into the Stern-Volmer equation:

$$
\frac{F_{0}}{F}=1+K_{\mathrm{SV}}[\mathrm{Q}]_{\mathrm{T}}
$$

where $F_{0}$ is the fluorescence intensity in the absence of quencher and $K_{\mathrm{SV}}$ is the Stern-Volmer constant, which is used as a measure of binding affinity in FQ experiments.

The violation of the assumptions (i-ii) may result in discrepancies between $K_{\mathrm{SV}}$ and true binding affinity $K_{\mathrm{A}}$ of the considered ligand-fluorophore system. First, the impact of equilibrium concentration of the complex [PQ] cannot be neglected for large binding constants, when significant part of ligand molecules is bound. This fact becomes especially important when using FQT for studying protein-drug binding, which is usually characterized by high affinity constants [16]. Also, derivation of the Stern-Volmer equation (3) implies that the quantum yield $\eta_{\text {complex }}$ of the complex is zero, but this is not the case for a number of systems.

Further, in this work it will be shown that based on multiple fluorescence quenching curves, measured at different total fluorophore concentration one may determine (a) if the number of binding sites in the system is equal to one or larger and (b) the quantum yield of the complex in the case of 1:1 stoichiometry.

\section{Materials and Methods}

\subsection{Samples preparation}

Bovine serum albumin (BSA), Congo Red (CR), 1-anilino-8-naphthalene sulfonate (ANS), sodium myristate (myr) and thioflavin T (ThT) were purchased in Sigma-Aldrich (Germany) and used without further purification. BSA stock solutions were prepared in phosphate buffered saline (PBS, $0.1 \mathrm{M}$ ionic strength) at pH 7.4. To obtain fluorescence quenching curves, stock solutions of Congo Red, ANS, and ThT dyes at a concentration of $100 \mu \mathrm{M}$ in PBS were prepared, and small volumes of obtained stock solutions were added to the solution with fixed protein concentration.

\subsection{Fluorescence measurements}

Fluorescence spectra were measured using the FluoroMax-4 (Horiba Jobin Yvon) spectrofluorometer. To avoid the inner-filter effect excitation wavelength 
was set to $295 \mathrm{~nm}$ that corresponds to the red edge of Trp absorption. Fluorescence emission was measured in the $325-400 \mathrm{~nm}$ range that corresponds to the fluorescence of tryptophan residues in BSA. Integral fluorescence values $F$ were calculated as the sum of fluorescence intensity signal in the $325-400 \mathrm{~nm}$ spectral range. Changes in fluorescence intensity $F$ due to changes in protein concentration upon titrant addition were corrected for all measurements.

Numerical simulation and experimental data processing were performed using the Python programming language and NumPy, SciPy, Matplotlib libraries. The online service for automatic data processing is available at http://lid.phys.msu.ru/ fluorescence-quenching/.

\section{Results and Discussion}

\subsection{Test on the difference between total and equilibrium concentration of the quencher}

To take into account that the total quencher concentration is not equal to its equilibrium concentration, one should use nonlinear fitting procedure and modified Stern-Volmer equation [7], which takes into account the assumptions on residuals distribution implied in least-squares procedure [17]. However, an alternative correction procedure that could be applied to experimental data to visually inspect
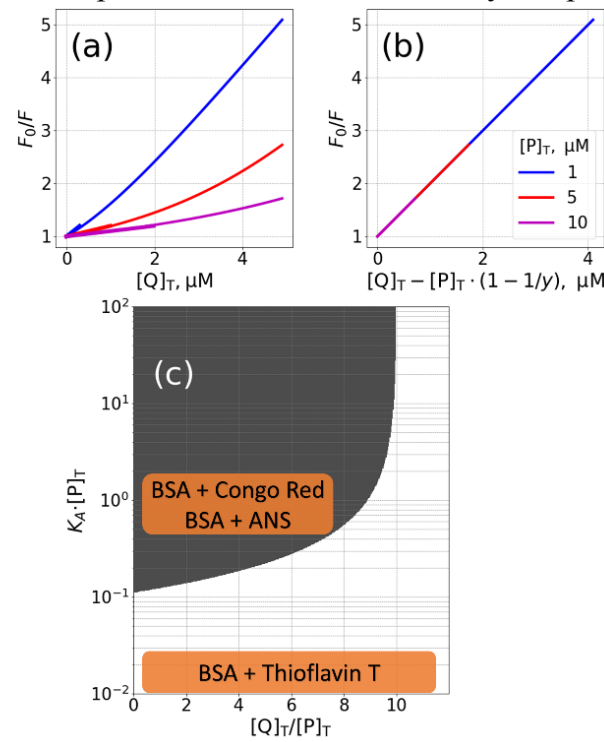

Fig. 1 (a) The FQ curves calculated for $K_{\mathrm{A}}=10^{6} \mathrm{M}^{-1}$ and different $[\mathrm{P}]_{\mathrm{T}}$ using Eq. (2). (b) FQ curves from Fig. 1a corrected for the difference between total and equilibrium concentration of quencher by plotting in the $\left([\mathrm{Q}]_{\mathrm{T}}-[\mathrm{P}]_{\mathrm{T}}\left(1-\frac{1}{y}\right), y\right)$ coordinates. (c) Diagram of $1: 1$ stoichiometry system parameters $\left(K_{\mathrm{A}} \cdot[\mathrm{P}]_{\mathrm{T}},[\mathrm{Q}]_{\mathrm{T}} /[\mathrm{P}]_{\mathrm{T}}\right)$, where correction for the difference between total and equilibrium concentration of quencher should (gray area) and should not (white area) be considered. The gray area corresponds to $\frac{[Q]}{[Q]_{\mathrm{T}}}<0.9$, where the assumption $[\mathrm{Q}] \approx[\mathrm{Q}]_{\mathrm{T}}$ is invalid. The orange areas correspond to parameters of the systems measured in this work. (d)-(e) FQ curves for BSA-CR before (d) and after (e) the correction procedure. BSA-CR FQ curves obtained for different $[\mathrm{P}]_{\mathrm{T}}$ without correction do not coincide due to significant difference between total and equilibrium concentration of quencher. Correction resulted in the coincidence of two FQ curves measured at different $[\mathrm{P}]_{\mathrm{T}}$. $(\mathrm{f})-(\mathrm{g})$. FQ curves for BSA-ThT before (f) and after $(\mathrm{g})$ correction procedure. Correction does not affect the FQ curves significantly due to low affinity constant in this system. whether the impact of the difference between total and equilibrium concentration is significant could benefit the data quality evaluation.

The equilibrium concentration could be derived using the following expression:

$$
[\mathrm{Q}]=[\mathrm{Q}]_{\mathrm{T}}-[\mathrm{PQ}]=[\mathrm{Q}]_{\mathrm{T}}-[\mathrm{P}]_{\mathrm{T}}\left(1-\frac{[\mathrm{P}]}{[\mathrm{P}]_{\mathrm{T}}}\right) .
$$

Still assuming that the quantum yield of complex equals zero, Eq. (3) can be rewritten as

$$
y=1+K_{\mathrm{A}}\left([\mathrm{Q}]_{\mathrm{T}}-[\mathrm{P}]_{\mathrm{T}}\left(1-\frac{1}{y}\right)\right)
$$

where $y=F_{0} / F$. Plotting the same FQ curves in the new coordinates $\left(y\right.$ over $[\mathrm{Q}]_{\mathrm{T}}-[\mathrm{P}]_{\mathrm{T}}\left(1-\frac{1}{y}\right)$ instead of of FQ plots caused by differences between total and equilibrium concentrations of quencher $\mathrm{Q}$. The nonmodified FQ curves calculated for $K_{\mathrm{A}}=10^{6} \mathrm{M}^{-1}$ and different $[\mathrm{P}]_{\mathrm{T}}$ are presented in Fig. 1a, where significant difference in their slope is clearly seen. This fact is due to violation of the assumption (i) at high $[\mathrm{P}]_{\mathrm{T}}$, while the proposed correction yields coinciding lines with the slope equal to $K_{\mathrm{A}}$ (Fig 1b). $[Q]_{T}$ ) thus should result in the correction of nonlinearity
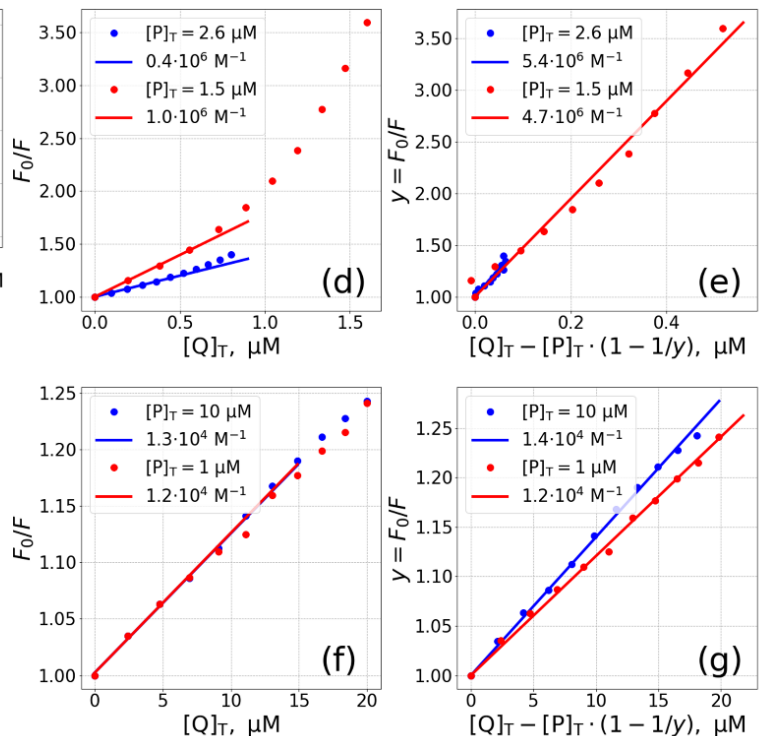
As the criterion for significant difference between [Q] and $[\mathrm{Q}]_{\mathrm{T}}$ we estimated parameter values of the system where $\frac{[\mathrm{Q}]}{[\mathrm{Q}]_{\mathrm{T}}} \leq 0.9$ (i.e. $[\mathrm{PQ}]>10 \%$ of $[\mathrm{Q}]_{\mathrm{T}}$ ). The results are presented in Fig. 1c, where the gray area corresponds to "forbidden" zone, meaning that at the corresponding parameters the assumption that $[\mathrm{Q}] \approx[\mathrm{Q}]_{\mathrm{T}}$ is violated and the affinity constant determined from Eq. (3) will be estimated with a high error.

To illustrate the importance of accounting for $[\mathrm{Q}] \neq[\mathrm{Q}]_{\mathrm{T}}$, we also performed experiments for model systems with high and low affinity constants: (a) BSA and Congo Red, (b) BSA and ThT. One more example with the BSA-ANS system is presented in the text below (Section 4.3). The BSA-CR system has $K_{\mathrm{A}} \sim 10^{6} \mathrm{M}^{-1}[17,18]$, hence, for the total protein concentration varying in range of $1-10 \mu \mathrm{M}$ the difference between equilibrium and total concentration should be taken into account. While fluorescence quenching curves for different $[\mathrm{P}]_{\mathrm{T}}$ differed significantly (Fig. 1d), correction for $[\mathrm{Q}] \neq[\mathrm{Q}]_{\mathrm{T}}$ using Eq. (5) resulted in their coincidence (Fig. 1e). The slopes of fluorescence quenching curves (i.e. binding affinities) obtained from the non-corrected SternVolmer plots (Fig. 1d) varied by an order of magnitude for different protein concentration $\left(4 \times 10^{5} \mathrm{M}^{-1}\right.$ for $[\mathrm{P}]_{\mathrm{T}}=2.6 \mu \mathrm{M}$ vs. $10^{6} \mathrm{M}^{-1}$ for $\left.[\mathrm{P}]_{\mathrm{T}}=1.5 \mu \mathrm{M}\right)$, while for the corrected plots yielded binding affinities of $\sim 5 \cdot 10^{6} \mathrm{M}^{-1}$ both for $[\mathrm{P}]_{\mathrm{T}}=1.5 \mu \mathrm{M}$ and $[\mathrm{P}]_{\mathrm{T}}=2.6 \mu \mathrm{M}$, respectively. Similar correction for the BSA-ThT system had no effect (Fig. 1f $-\mathrm{g}$ ) because of low binding affinity in this system [18].

\subsection{Assessment of fluorescence quantum yield of a protein-ligand complex}

Derivation of the SV equation (3) also implies that the quantum yield $\eta_{\text {complex }}$ of the complex is zero, i.e. that the quenching is complete. As this assumption is violated in a number of systems, the question is whether it is possible to prove that $\eta_{\text {complex }}$ is non-zero. The ratio $F_{0} / F$ for the $1: 1$ complexation in case of non-zero quantum yield can be expressed as:

$$
\begin{gathered}
\frac{F_{0}}{F}=\frac{\eta_{\text {Protein }}[\mathrm{P}]_{\mathrm{T}}}{\eta_{\text {Protein }}[\mathrm{P}]+\eta_{\mathrm{PQ}}[\mathrm{PQ}]}= \\
=\frac{[\mathrm{P}]_{\mathrm{T}}}{[\mathrm{P}]+\eta[\mathrm{PQ}]}=\frac{[\mathrm{P}]_{\mathrm{T}}}{[\mathrm{P}]_{\mathrm{T}}+(\eta-1)[\mathrm{PQ}]},
\end{gathered}
$$

where $\eta \equiv \frac{\eta_{\text {complex }}}{\eta_{\text {Protein }}}$ is the relative quantum yield of the complex $(0 \leq \eta<1)$. By measuring $y=\frac{F_{0}}{F}$ it is possible to estimate the value of $[\mathrm{PQ}]$ :

$$
y=\frac{[\mathrm{P}]_{\mathrm{T}}}{[\mathrm{P}]_{\mathrm{T}}+(\eta-1)[\mathrm{PQ}]} \Rightarrow[\mathrm{PQ}]=\frac{[\mathrm{P}]_{\mathrm{T}}\left(1-\frac{1}{y}\right)}{1-\eta} .
$$

Concentration of free quencher can be expressed as:

$$
[\mathrm{Q}]=[\mathrm{Q}]_{\mathrm{T}}-[\mathrm{PQ}]=[\mathrm{Q}]_{\mathrm{T}}-\frac{[\mathrm{P}]_{\mathrm{T}}\left(1-\frac{1}{y}\right)}{1-\eta}
$$

Next, combining Eq. (2) and Eq. (7) yields:

$$
y=\frac{F_{0}}{F}=\frac{[\mathrm{P}]_{\mathrm{T}}}{[\mathrm{P}]+\eta[\mathrm{PQ}]}=\frac{1+\mathrm{K}_{\mathrm{A}}[\mathrm{Q}]}{1+\eta \mathrm{K}_{\mathrm{A}}[\mathrm{Q}]} .
$$

Finally, by substituting the Eq. (8) into Eq. (9), one may obtain the following parametric equation:

$$
\frac{y-1}{1-\eta y}=K_{\mathrm{A}}\left([\mathrm{Q}]_{\mathrm{T}}-[\mathrm{P}]_{\mathrm{T}} \frac{1-\frac{1}{y}}{1-\eta}\right) .
$$

Eq. (10) describes the dependence of fluorescence quenching ratio $F_{0} / F$ on total concentration of fluorophore $[\mathrm{P}]_{\mathrm{T}}$ and quencher $[\mathrm{Q}]_{\mathrm{T}}$ without the assumptions $[\mathrm{Q}] \approx[\mathrm{Q}]_{\mathrm{T}}$ and $\eta_{\text {complex }} \approx 0$. To determine the values of $\eta$ and $K_{\mathrm{A}}$, we suggest to measure at least two $F Q$ for different $[\mathrm{P}]_{\mathrm{T}}$, and then simultaneously fit these curves to Eq. (10) to obtain the $\eta$ and $K_{\mathrm{A}}$ parameters. The main idea of this approach is that the Eq. (10) allows to consider the $y$ values as function $y=y\left([\mathrm{Q}]_{\mathrm{T}},[\mathrm{P}]_{\mathrm{T}}, K_{\mathrm{A}}, \eta\right)$ with two independent variables $[\mathrm{Q}]_{\mathrm{T}},[\mathrm{P}]_{\mathrm{T}}$ and two parameters $K_{\mathrm{A}}, \eta$, thus the reliable evaluation of these parameters might be done.

The Eq. (10) could be also rewritten in the form suggested in Ref. [7]. Despite the correctness of the equations in Ref. [7], we found out that using a titration curve for a single concentration of protein could result in poor accuracy in determination of binding constant $K_{\mathrm{A}}$ and relative quantum yield $\eta$. To demonstrate this we modelled the fluorescence quenching curves for system with 1:1 stoichiometry and incomplete quenching with $K_{\mathrm{A}}=2 \times 10^{5} \mathrm{M}^{-1}$ and $\eta=0.3$ for two concentrations of protein $[\mathrm{P}]_{\mathrm{T}}=1 \mu \mathrm{M}$ and $[\mathrm{P}]_{\mathrm{T}}=10 \mu \mathrm{M}$ with total concentration of quencher $\mathrm{Q}$ varied in such region so the $F_{0} / F$ ratio was lower than 1.5 for both protein concentrations. It was found that the binding constant and relative quantum yield of complex system obtained at $[\mathrm{P}]_{\mathrm{T}}=1 \mu \mathrm{M}$ were equal to $K_{\mathrm{A}}=(1.53 \pm 0.21) \times 10^{6}$ and $\eta=0,15 \pm 0.08$, while for $[\mathrm{P}]_{\mathrm{T}}=10 \mu \mathrm{M}, K_{\mathrm{A}}=(9 \pm 4) \times 10^{5} \mathrm{M}^{-1}$ and $\eta=0,0001 \pm 0,0019$ were obtained. At the same time, simultaneous use of two titration curves modelled for two concentrations of protein resulted in $K_{\mathrm{A}}=(1.8+0.3) \times 10^{6} \mathrm{M}^{-1}$ and $\eta=0,26 \pm 0.02$ that coincided with the true values within the error. Hence, we emphasize that even using correct equation for analysing a single titration curve could be insufficient to obtain reliable results on binding parameters of the system, and using two titration curves is beneficial.

\subsection{Estimation of the number of binding sites}

Currently, in order to obtain the number of binding sites from fluorescence quenching curves, the "log-log" Stern Volmer equation is often used. However, it has been demonstrated by several authors that this approach is incorrect $[6-8,10]$. The question about the possibility to 
obtain the information about stoichiometry using fluorescence methods was reviewed in detail in Refs. $[14,15]$, and as it was already mentioned, for determination of the exact number of binding sites it is crucial to measure several titration curves with ligand concentrations up to the values that significantly exceed $1 / K_{A}[14,15]$. In this work we propose the method for simple FQT data processing in the region of parameters, where saturation is not achieved, to test the hypotheses whether a single binding site $(n=1)$ or multiple binding sites $(n>1)$ are present in the system. The procedure for this can be done as follows.

Step 1. One should measure fluorescence quenching curves for two (or more) titration series for different initial values of $[\mathrm{P}]_{\mathrm{T}}$. Theoretically, $[\mathrm{P}]_{\mathrm{T}}$ values should be chosen in the way that $K_{\mathrm{A}}[\mathrm{P}]_{\mathrm{T}}^{(1)} \ll 1$ and $K_{\mathrm{A}}[\mathrm{P}]_{\mathrm{T}}^{(2)} \sim 1$ as for at these values the FQ curves would differ significantly. At the same time, one should find a compromise between two experimental artifacts. On the one hand, low values of $[\mathrm{P}]_{\mathrm{T}}$ lead to low intensity signals. On the other hand, high values of $[\mathrm{P}]_{\mathrm{T}}$ lead to inner filter effect, which also should be taken into account. For example, for BSA these values can be suggested to be equal to $[\mathrm{P}]_{\mathrm{T}}^{(1)}=1 \mu \mathrm{M}$ and $[\mathrm{P}]_{\mathrm{T}}^{(2)}=10 \mu \mathrm{M}$. If the FQ curves measured at different $[\mathrm{P}]_{\mathrm{T}}$ values coincide, it can be argued that both assumptions of Stern-Volmer equation (i) and (ii) are fulfilled and further corrections are not necessary.

Step 2. If the FQ curves measured at two $[\mathrm{P}]_{\mathrm{T}}$ values differ, the procedure for fluorescence quantum yield estimation, i.e. fitting them to Eq. (10) should be performed. As a result, the values $K_{\mathrm{A}}, \eta$ will be obtained.

Step 3. If for both FQ curves $R^{2}>0.95$ and the residuals are not correlated (that could be verified visually or by using the Durbin-Watson statistical test) the fitting procedure can be considered successful. In this case, it can be argued that $n=1$ and the relative quantum yield of complex and binding affinity constant $K_{\mathrm{A}}$ are determined correctly. If $R^{2}<0.95$, or residuals are correlated, it can be concluded that the system is not described by Eq. (10) and $n>1$.

The described algorithm is visualized in Fig. 2.

To illustrate how this method works, the following systems were selected: the BSA-sodium myristate (myr) system, where $n>1[20,21]$; BSA-ANS, where it is known that number of binding sites is 2 and fluorescence of the complex is not completely quenched [22]; and BSA-Congo Red where $n=1[18,19]$. For all the systems two FQ curves were measured at different $[\mathrm{P}]_{\mathrm{T}}($ Fig. $3 \mathrm{a}-\mathrm{c})$, and then the suggested approach was applied. The $R^{2}$ value for the BSA-myr system was equal to 0.407 , there were clearly visible trends in residuals behavior (Fig. 3d), and the value of the Durbin-Watson's statistics was equal to 0.05 (the value of the Durbin-Watson statistic close to zero means the presence of positive correlation between residuals, a lack of correlation corresponds to the value of DurbinWatson statistic equal to 2). Hence, it was verified that $n>1$. Fit of the BSA-ANS data also exhibited poor $R^{2}=0.915$ value and correlated residuals (Fig. $3 \mathrm{e}$ ).

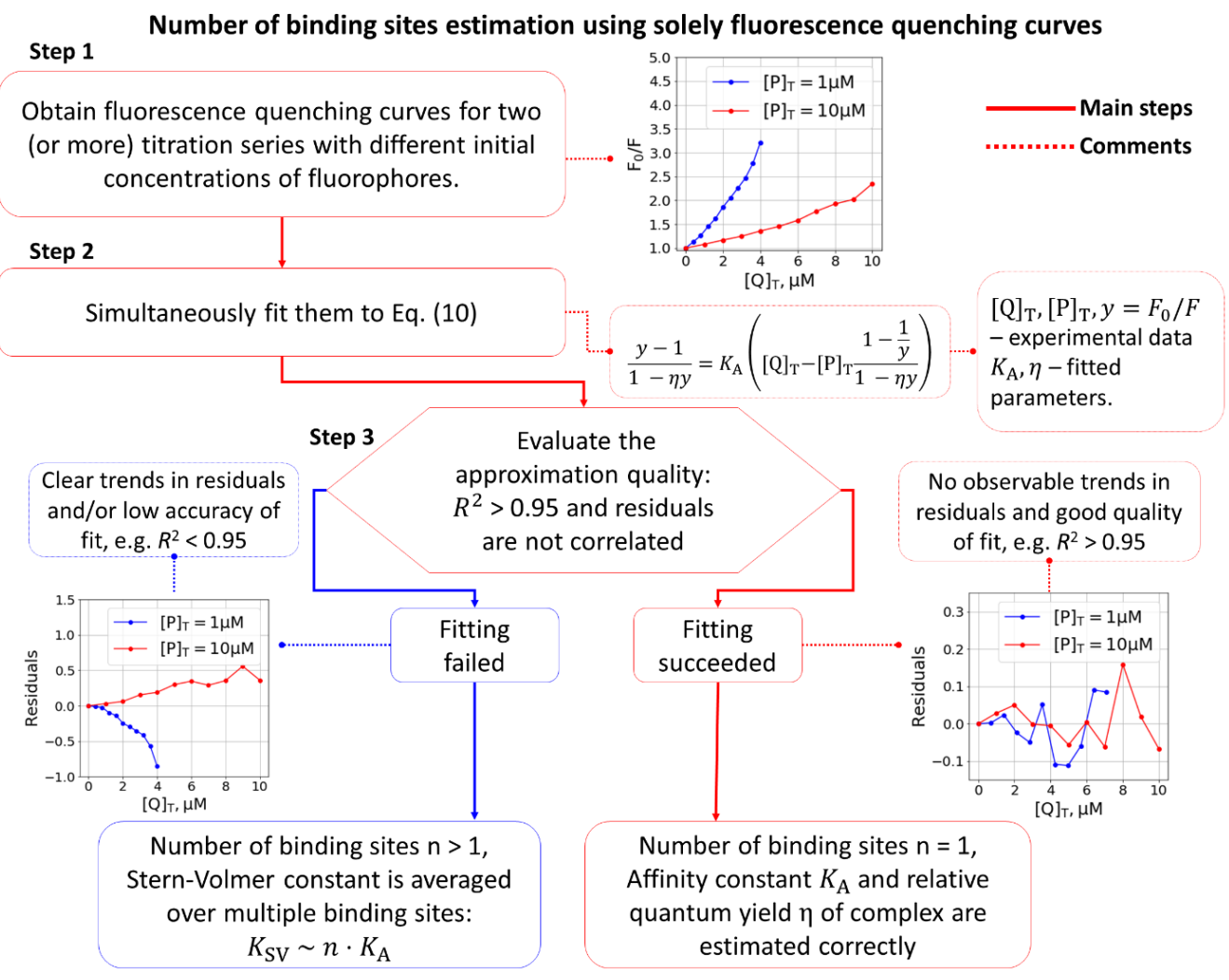

Fig. 2 Schematic representation of steps to be performed to analyze fluorescence quenching curves. 

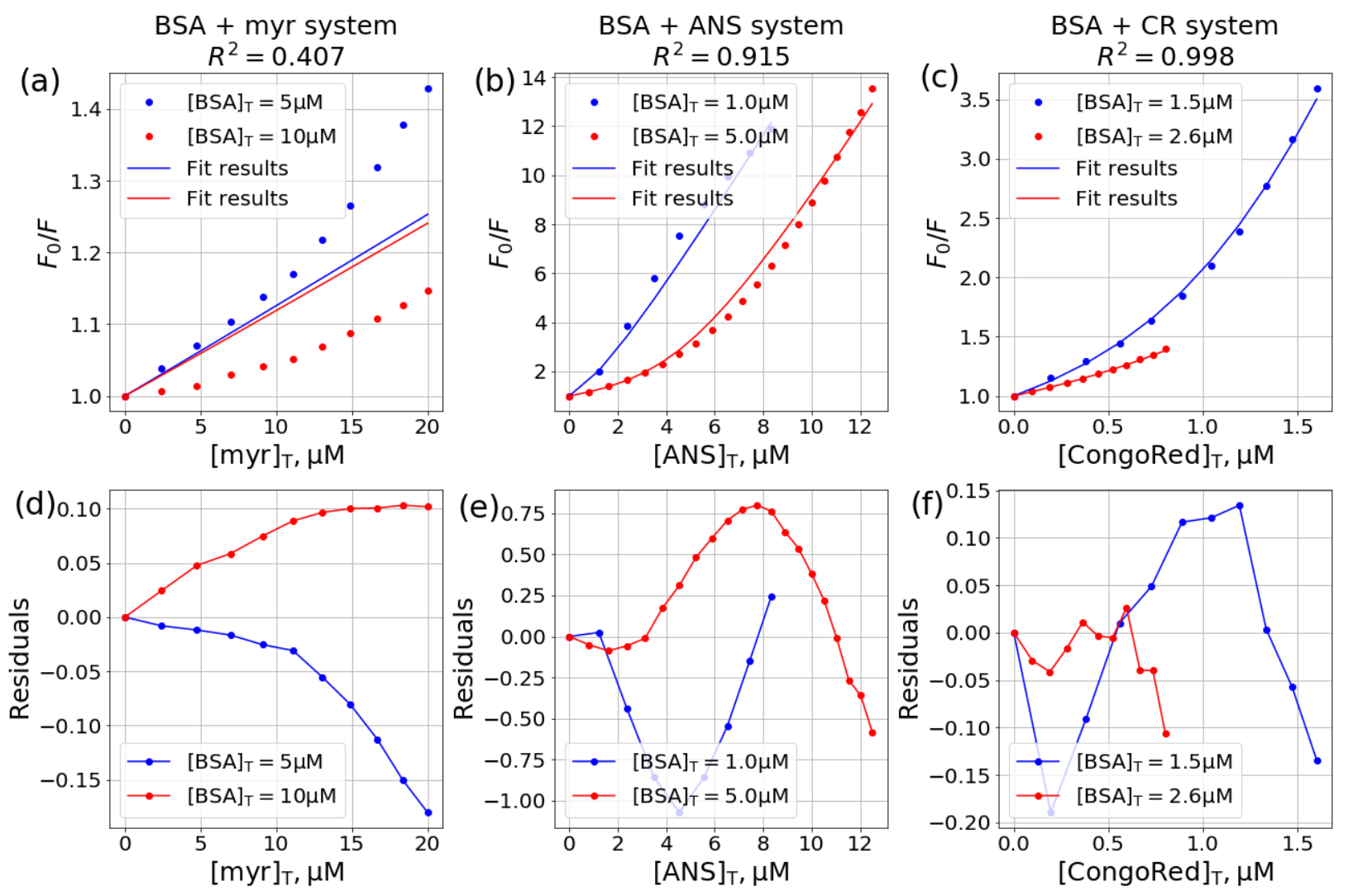

Fig. 3 (a)-(c) Fluorescence quenching curves for the BSA-myr (a), BSA-ANS (b) and BSA-CR (c) systems. (d)-(f) Residuals of fit of FQ curves by Eq. (10) for the BSA-myr (d), BSA-ANS (e), BSA-CR (f). Clearly observable trends in residuals may be noted for BSA-myr and BSA-ANS systems, verifying that number of binding sites is greater than 1 for these systems.

On the contrary, when the proposed algorithm was applied to the BSA-CR system, where $n=1$ (Fig. 3c), the $R^{2}$ value was equal to 0.998 (Fig. 3f), and the values of the Durbin-Watson's statistics were equal to 1.5. Hence, it can be concluded that $n=1$. The values obtained from fitting the FQ curves to Eq. (10) were $K_{\mathrm{A}}=(4.6 \pm 0.4) \times 10^{6} \mathrm{M}^{-1}$ and $\eta=0.003 \pm 0.018$, suggesting complete quenching in the system.

The suggested algorithm was also investigated numerically. Several systems were taken into consideration: (a) the system with one binding site and zero quantum yield of the complex, (b) the system with one binding site and non-zero quantum yield of the complex and (c) the system with several binding sites ( $n=2,3,4,5$ and $K_{\mathrm{A}_{i}}=\frac{K_{\mathrm{A}_{i-1}}}{2}$, zero quantum yields). For each system the series of FQ experiments (two FQ curves with $[\mathrm{P}]_{\mathrm{T}}^{(1)}=1 \mu \mathrm{M},[\mathrm{P}]_{\mathrm{T}}^{(2)}=10 \mu \mathrm{M}$ ) were simulated. To simulate the experimental error, five percent noise with uniform distribution was added to the $F$ values. Then the suggested method for estimation of $K_{\mathrm{A}}$ and quantum yield was applied to fit the simulated data. Overall, 10000 experiments were simulated for all parameters sets. Fig. 4 demonstrates the contingency matrices of algorithm predictions for the system with low and high affinities $\left(K_{\mathrm{A}}=5 \times 10^{4} \mathrm{M}^{-1}\right.$ and $K_{\mathrm{A}}=5 \times 10^{6} \mathrm{M}^{-1}$, respectively). For the high affinity systems the algorithm successfully distinguished three cases: the 1:1 stoichiometry with zero quantum yield of complex, 1:1 complexation with non-zero quantum yield and the $n>1$ case, yielding almost $100 \%$ accuracy. Higher error rates were observed for low affinity systems: classification algorithm was prone to falsely attribute $n=1$ systems with non-zero quantum yield to systems with $n>1$. However, firstly, the case of high affinities is of more interest for biochemistry and pharmacology, and, secondly, as it was demonstrated in the section 4.1, FQ curves measured at different $[\mathrm{P}]_{\mathrm{T}}$ for low affinity constants coincide before the correction procedure, so one may classify the case of low binding affinities without applying the suggested algorithm.

\subsection{Web-interface for the $F Q$ data processing}

To simplify the data processing using the suggested approach, the online service for data processing has been developed. (link: http://lid.phys.msu.ru/ fluorescence-quenching/). Users can upload their data (two FQ curves), as a result, the procedure application summary is displayed. At first, the cases of $n=1$ and $n>1$ are discriminated; then in case of $n=1$, $K_{\mathrm{A}}, \eta, R^{2}$ values and the values of Durbin-Watson's statistics are shown; in case of $n>1$ the $R^{2}$ value and the values of Durbin-Watson's statistics are displayed. 
data, residuals and fits to an Eq. (10) with obtained parameters are presented in plots. Accurate estimation the number of binding sites, affinity constants and quantum yields of each of binding sites cannot be performed using solely fluorescence quenching method without any additional assumptions and restrictions imposed on quantum yields and binding affinities. So other experimental techniques should be used in addition to FQ technique to determine system properties.
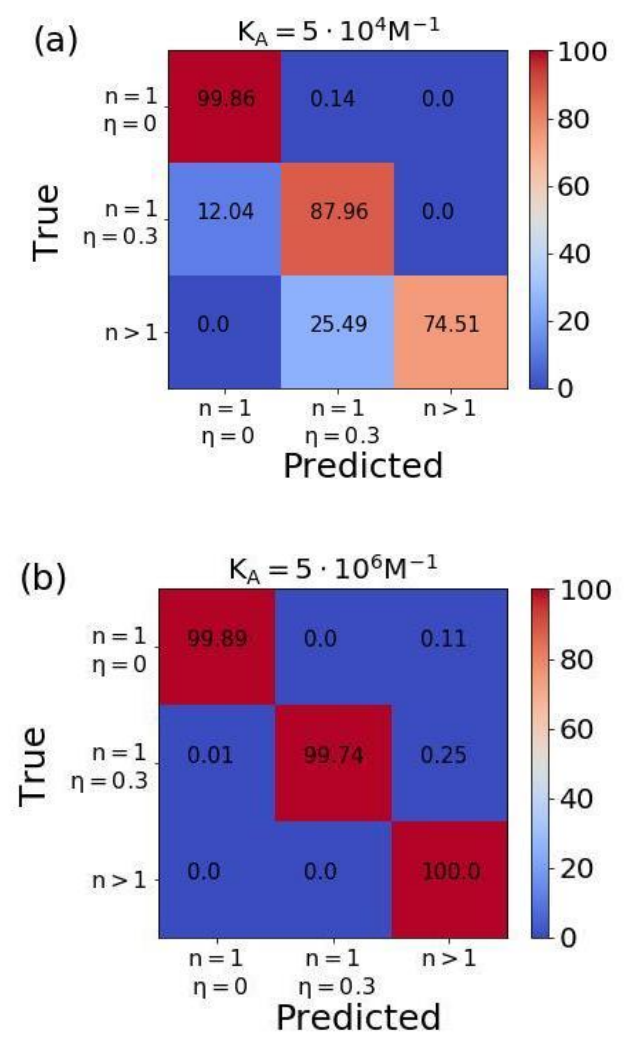

Fig. 4 The results of predictions of the proposed algorithm presented in the form of contingency matrices. Percentage of predicted vs. true parameters of the modeled FQ experiments is presented in each cell. (a) The contingency matrix for the system with low affinity $\left(K_{\mathrm{A}}=5 \times 10^{4} \mathrm{M}^{-1}\right)$. b) The contingency matrix for $K_{\mathrm{A}}=5 \times 10^{6} \mathrm{M}^{-1}$. It can be noted that the proposed algorithm is better suited for higher values of affinity constants commonly occurring in biochemistry and pharmacology.

\section{Conclusion}

A number of papers has been devoted to the discussion of fluorescence quenching technique for binding parameters estimation. In this paper, we focused on the case of tryptophan fluorescence quenching in proteins upon binding and tried to suggest a simple algorithm for FQT data processing for the curves measured at typical experimental conditions. As some of the authors before [14-15], we came to the conclusion that measuring more than one fluorescence quenching curve is highly advantageous. That is, even application of the established procedure for assessing the fluorescence quantum yield [7] to a single curve may lead to discrepancy in the determined parameters. On the other hand, the use of some advanced algorithms [14-15] requires reaching a saturation in quenching, i.e. the use of high ligand concentrations, that has certain shortcomings - e.g. high optical density. Here we considered the application of the fluorescence quenching method to determine binding constant, number of binding sites and quantum yield of complex under the most typical experimental conditions. We demonstrated, both theoretically and experimentally, that several procedures can be applied to avoid common pitfalls that are encountered in processing and interpretation of FQ curves. A method was proposed that allows one to estimate the number of binding sites and the relative quantum yield of the complex in case of $1: 1$ stoichiometry. It was shown that in the case of high affinity constants $\left(\sim 10^{6} \mathrm{M}^{-1}\right)$, the presence of several binding sites can be determined by measuring multiple FQ curves with different initial fluorophore concentrations. Measurement of multiple fluorescence quenching curves is necessary to reliably estimate the effect of differences in the equilibrium and total quencher concentration and to estimate the complex quantum yield. We also present the web-interface for automated processing of fluorescence quenching experiments based on the suggested approach.

\section{Disclosures}

All authors declare that there is no conflict of interests in this paper.

\section{References}

1. M. E. Burton, L. M. Shaw, J. J. Schentag, and W. E. Evans (eds.), Applied pharmacokinetics \& pharmacodynamics: principles of therapeutic drug monitoring, Lippincott Williams \& Wilkins, Philadelphia, USA (2006).

2. D. L. Nelson, A. Lehninger, and M. M. Cox, Lehninger - principles of biochemistry, D. Nelson, M. B. Cox (Eds.), Macmillan, London, UK (2008).

3. K. Hirose, "A practical guide for the determination of binding constants," Journal of inclusion phenomena and macrocyclic chemistry 39(3), 193-209 (2001). 
4. J. R. Lakowicz (Ed.), Principles of fluorescence spectroscopy, Springer Science \& Business Media, Berlin/Heidelberg, Germany (2013).

5. P. R. Callis, "Binding phenomena and fluorescence quenching. I: descriptive quantum principles of fluorescence quenching using a supermolecule approach," Journal of Molecular Structure 1077, 14-21 (2014).

6. M. Van De Weert, "Fluorescence quenching to study protein-ligand binding: common errors," Journal of fluorescence 20(2), 625-629 (2010).

7. M. Van de Weert, L. Stella, "Fluorescence quenching and ligand binding: A critical discussion of a popular methodology," Journal of Molecular Structure 998(1-3), 144-150 (2011).

8. E. Lissi, C. Calderón, and A. Campos, "Evaluation of the number of binding sites in proteins from their intrinsic fluorescence: limitations and pitfalls," Photochemistry and photobiology 89(6), 1413-1416 (2013).

9. T. N. Tikhonova, E. A. Shirshin, G. S. Budylin, V. V. Fadeev, and G. P. Petrova, "Assessment of the europium(III) binding sites on albumin using fluorescence spectroscopy," The Journal of Physical Chemistry B 118(240), 66266633 (2014).

10. M. van de Weert, L Stella, "The dangers of citing papers you did not read or understand," Journal of Molecular Structure 1186, 102-103 (2019).

11. Y. Hong, J. W. Lam, and B. Z. Tang, “Aggregation-induced emission,” Chemical Society Reviews 40(11), 53615388 (2011).

12. M. R. Eftink, Fluorescence methods for studying equilibrium macromolecule-ligand interactions, In Methods in enzymology 278, 221-225 (1997).

13. L. D. Ward, Measurement of ligand binding to proteins by fluorescence spectroscopy, Chapter in Methods in enzymology 117, 400-414 (1985).

14. W. Bujalowski, M. J. Jezewska, and P. J. Bujalowski, "Signal and binding. I. Physico-chemical response to macromolecule-ligand interactions," Biophysical Chemistry 222, 7-24 (2017).

15. W. Bujalowski, M. J. Jezewska, and P. J. Bujalowski, "Signal and binding. II. Converting physico-chemical responses to macromoleculeligand interactions into thermodynamic binding isotherms," Biophysical Chemistry 222, 25-40 (2017).

16. V. Kairys, L. Baranauskiene, M. Kazlauskiene, D. Matulis, and E. Kazlauskas, "Binding affinity in drug design: experimental and computational techniques," Expert opinion on drug discovery 14(8), 755-768 (2019).

17. R. J. Leatherbarrow, "Using linear and non-linear regression to fit biochemical data," Trends in biochemical sciences 15(12), 455-458 (1990).

18. Y.-Z. Zhang, X. Xiang, P. Mei, J. Dai, L.-L. Zhang, and Y. Liu, "Spectroscopic studies on the interaction of Congo Red with bovine serum albumin," Spectrochimica Acta Part A: Molecular and Biomolecular Spectroscopy 72(4), 907-914 (2009).

19. H. M. C. de Paula, Y. L. Coelho, A. J. P. Agudelo, J. de Paula Rezende, G. M. D. Ferreira, G. M. D. Ferreira, A. C. dos Santos Pires, and L. H. M. da Silva, "Kinetics and thermodynamics of bovine serum albumin interactions with Congo red dye," Colloids and Surfaces B: Biointerfaces 159, 737-742 (2017).

20. J. A. Hamilton, D. P. Cistola, J. D. Morrisett, J. T. Sparrow, and D. M. Small, "Interactions of myristic acid with bovine serum albumin: a 13C NMR study," Proceedings of the National Academy of Sciences 81(12), 3718-3722 (1984).

21. E. L. Gelamo, C. H. T. P. Silva, H. Imasato, and M. Tabak, "Interaction of bovine (BSA) and human (HSA) serum albumins with ionic surfactants: spectroscopy and modelling," Biochimica et Biophysica Acta (BBA)-Protein Structure and Molecular Enzymology 1594(1), 84-99 (2002).

22. I. M. Kuznetsova, A. I. Sulatskaya, O. I. Povarova, and K. K. Turoverov, "Reevaluation of ANS binding to human and bovine serum albumins: key role of equilibrium microdialysis in ligand-receptor binding characterization," PLoS One 7(7), e40845 (2012). 